\title{
LL explorations and methodological challenges
}

\section{Analysing France's regional languages}

\section{Robert Blackwood}

University of Liverpool

The methodologies employed over these first years of LL research have evolved rapidly in several different directions, although quantitative and/or qualitative approaches have guided most published scholarship thus far. The quantitative approach has come to be reduced in one particular narrative to the counting of signs, whilst qualitative research is portrayed as permitting analysis of a selection of signs from which wider conclusions can be drawn. Using an on-going project into France's regional languages in the LL, this article argues that a symbiotic approach is essential for contributing to discussions on language revitalization in the public space. Whilst quantitative data collection contextualizes language use, a subsequent qualitative examination, along several vectors, avoids impressionistic conclusions about the correlation between visibility and vitality. We contend here that this dual approach permits cross-referencing across space and time in ways not possible by adopting one or other methodology on its own.

Keywords: quantitative, qualitative, methodologies, France, regional language, revitalization, diachronic

\section{Introduction}

Since the summer of 2007, not long after the publication of Gorter's 2006 collection of essays on multilingualism in the LL, I have been exploring the written varieties of France's regional languages (RLs) in the public space. Although this project is still on-going, eight years since my first LL fieldwork, the launch of this new journal is a great opportunity to take stock, to reflect on developments in LL research, to consider critically the shortcomings in my own work, and to seek to contribute to wider debates, all through the prism of the visibility of France's RLs on the walls of cities, towns, and villages across France. By France, we refer 
to the widest possible understanding of territories identified with France, hence the inclusion in my fieldwork of two islands in the Caribbean and one collectivity of islands in the South Pacific, as well as parts of European France. This project has been premised on the understanding that language visibility in the public space comments in some way on language vitality. Landry and Bourhis (1997:28) contend that 'the presence or absence of rival languages in specific domains of the linguistic landscape can come to symbolize the strength or weakness of competing groups', a position that Ben-Rafael, Shohamy, Amara, and Trumper-Hecht (2006:7) nuance further, noting that languages define the 'symbolic construction of the public space'. Although Sáez Rivera and Castillo Lluch (2012:316) suggest ethnolinguistic vitality underpins both presence and longevity in the LL, we contend, along with Barni and Vedovelli (2012:28) that there is no direct correlation between the visibility of a language in the LL and its vitality. It seems highly implausible that that those who manage the public space necessarily seek to do so exactly in accordance with the language practices of a given community - indeed, it is pertinent to ask how we are ever able to ascertain accurately the languages that groups and individuals speak at any given time in order to reflect this on the walls of the towns where they live.

Instead, we venture that the presence of a regional language in a city identified to some extent with an ethnolinguistic community says something about language beliefs towards that language. In other words, the extent to which a language is visible is one approach to understanding better attitudes towards that language. Part of the work of the LL researcher is to analyse and interpret this presence or absence with a view to reaching a judgment on the extent to which a language is used, valued, or rejected by managers of the public space. However, the data (and their collection) with which the researcher engages are not something upon which there is widespread agreement, and, using France as the context, we propose here to examine the methodological challenges that have emerged when examining the relationship between a seemingly unequivocal language policy and language attitudes towards long-established RLs. LL data collection is increasingly framed as a dialectic of quantitative and qualitative approaches, and we explore the extent to which this division is both unhelpful and reductive. We aim, therefore, to discuss the benefits of a synergetic approach to data collection which does not merely provide two different methods to scrutinize the same landscape, but instead combines distinct techniques which, together, provide a fuller, more comprehensive understanding of language revitalization, commodification, and the reversal of a language shift.

At the outset of this debate, it is important to highlight that quantitative research has always been at the heart of this project, and has consistently been the starting point for this fieldwork in France. In recent years, there has been a 
reductivist tendency to simplify the quantitative approach as merely involving the counting of signs, whereas qualitative research is viewed more positively; it explores issues pertaining to the behaviour of the social actor, using the LL as a site of inquiry. Clearly, this is a simplified description of the two methodologies, but in this section we seek to outline ways by which the quantitative approach is a prerequisite for LL research which seeks to describe and analyse a given space. At the same time, questions of authorship, first raised by Malinowski (2009), and the debate surrounding social circulation of languages, addressed by Stroud and Mpendukana (2009), have brought into sharp focus the imperative to look beyond the surface form of the sign (however a given researcher understands a 'sign') to explore contexts, constructs, and connections. As such, we argue that a symbiotic approach, where the quantitative and qualitative approaches feed into one another, is an ideal modus operandi.

\section{Methodological challenges}

For this longitudinal project on France's RLs, by a quantitative approach, we refer not only to the establishing of a clear survey area and the counting of signs, but also their careful coding along several matrices, including their function, distribution, language configuration, and frequency. The statistics generated by this approach to data collection are subsequently analysed and findings scrutinized. At the earliest meetings where the LL was debated, a significant proportion of the discussion focussed on the fundamentals of quantitative approaches. Much time was initially devoted to understanding the nature of a sign, and how the definition of a sign plays a significant part in the understanding of language in the public space. Nowadays, many of those undertaking quantitative research - including this project - adopt the definition proposed by Backhaus (2007:66) 'any piece of text within a spatially definable frame' as a workable concept. From this, we do not infer that the question of the nature of the sign is resolved, but rather that the focus of the debate has moved on without a broad consensus. Huebner, an early adopter of the LL, raised this question in particular. He highlighted that the most straightforward definition failed to differentiate between the sizes of a sign. In his words (2009:71), '[...] the resulting analyses afford equal weight to a $3 \times 6$ inch sign reading "pull" adjacent to the handle of a shop door, to a $2 \times 5$ foot banner hanging from a light pole advertising a movie, and to a $20 \times 40$ foot sign proclaiming the name, telephone number and products of the shop itself'. The point Huebner raised has not really been addressed by those looking quantitatively at the LL, and for the time being, this challenge remains unresolved. It is entirely plausible that there is a logarithm by which the relative size and position of a sign can be taken 
into account when considering its visual impact, but its complexity is such that it is yet to be devised, let alone built into analyses of language in the public space. The various competing pressures, and the extent to which experience of what people see (and do not see) is so individualized that devising an accurate and widely applicable model appears particularly challenging.

At the same time that the debate around the nature of the sign has not been resolved, the choice of a survey area remains problematic. It is challenging to the point of being unfeasible to survey an entire city or town; of course, technological advances might well mean that, in the coming years, it will be possible to undertake an authoritative, comprehensive study of all the public space in a given city. Practically, and certainly over the lifespan of this project, no definitive answer has been found as to how to define a practical survey area when seeking to measure the written use of regional languages in parts of France. As set out elsewhere (Blackwood, 2010), survey areas for this project have been identified on the basis of what has been perceived, on the ground at the outset of the data collection, to be the main streets of the survey city. Subsequently, a starting point from which to measure out a fifty-metre stretch has been selected arbitrarily. This approach facilitates the research, but what it does not do is remove the scope for criticism of the methodology. For those undertaking quantitative LL research, this is another point that has been raised but not addressed in any definitive kind of way, in part because the focus of the debate has shifted, and in part because there is no obvious solution.

The coding of signs was the other key point raised by Gorter in 2006. For this project on France's regional languages, we adapted a nine-variable coding system, premised on the function that the sign performs. These are 1) business names, 2) business signs, 3) graffiti, 4) information, 5) instructions, 6) labels on products, 7) legends (which includes slogans, trades association membership stickers, etc), 8) street names, 9) trademarks. The flaws in this typology have emerged over the six years of fieldwork in France, although it was clear at the outset that there is the potential for tension between these functions, especially when signs can fulfil different roles simultaneously. This shortcoming is one that has endured, and the coding process has been premised on what is perceived to be the main function of a sign, although this continues to be based on the evaluation made at the time of coding. These are the categories that we continue to use, although in some cases, we would refine some of the categories, collapse others together, and divide some up into other codes. For example, in Nice with Monaco, in a corpus of 11, 327 signs, only four are recorded as graffiti, whilst 62 are street signs, and 190 are instructions. It might well be that this distribution accurately reflects the composition of the written text in the public space, but proportionally, these three codings barely register in a quantitative study, something which we argue highlights the 
challenges behind this typology. In terms of these three marginal functions, graffiti is the category that seems least practical. One explanation for this might be that a notional golden age of graffiti on the walls of France, where political and social commentaries were played out in the LL, has long been deserted in favour of a sprinkling of tags, the occasional declaration of love or hatred, and rare partisan outbursts. Moreover, on the basis of this project, graffiti, especially tags, are rarely part of language revitalization efforts. Milon (2002:88) defines a tag as 'a more or less complex monochromatic mural signature', whose relation to the focus of this project, we argue, is at best tangential. Moreover, according to Pennycook (2010:60) graffiti is not 'generally aimed at easy public consumption' and therefore unlikely to contribute to the wider public debate on the use of France's regional languages.

Street signs play a significant role in the exploration of the LL since, from early on in this kind of research, they have been emblematic of multilingualism in the public space whilst, simultaneously, achieving prominence in the eyes of the wider public. In France, as elsewhere, debates on the use of languages in street signs have taken place on the public stage, in forums including newspaper editorials, letters pages, on-line discussion groups, and social media platforms. In part, the debate centres on the official use of RLs (with their contested statuses), where regional activists clash with devout French Republicans cherishing the national standard language. As Amos (forthcoming) notes, the street sign is 'not only a marker of top-down language policy, but also an active component in the construction of identity, and of the perceptions [...] of the authority's management of the languages it oversees'. Although numerically a category of marginal significance, and although street names and - especially — road signs often appear in liminal spaces, their peripherality in physical and quantitative terms is in opposition to their prominence in the public eye, and their centrality to long-standing local debates about multilingualism in France. For these reasons, we argue that they earn their place in a modern typology of the LL.

At the other end of the scale, the category 'information' simply does not offer the level of granularity required to permit close analysis of the language choices in texts in the LL. Of the 3990 signs coded as information in Nice with Monaco in 2011, a second level of codification has emerged ad hoc, including details such as 'services offered', or 'menu', or 'price list' or 'poster'. Having established the initial methodology for this project, we explore in the next sections the possibilities and shortcomings that emerge from a quantitative approach, and to illustrate this point, we will focus in particular on the findings from the surveys in Brittany, Corsica, and Northern Catalonia, with occasional reference to the other investigations. 


\section{Challenges and opportunities of quantitative approaches}

This project, having surveyed nine areas across France, each focussing on a different RL, highlights the challenges and opportunities of both methods of data collection, and in this section we identify those issues presented by the quantitative collection approach, specifically from the perspective of language revitalization. The limitations of this methodology highlight in particular the necessity of a combined approach when seeking to explore language revitalization, upon which we focus in the next section.

\subsection{The benefits of a quantitative approach}

\subsubsection{Comparability}

Thus far, fieldwork has been undertaken in Brittany (2007), Corsica (2007), Northern Catalonia (2008), Marseille (2008), Flemish France (2009), Guadeloupe (2010), Martinique (2010), Nice with Monaco (2011), and New Caledonia (2013), all according to the same methodology outlined in Section 2 above. This project covers a vast geographical space and its conclusions, especially those which seek to differentiate between the vitality of different languages within the same State, are premised on the basis that the data collection is robust and identical (insofar as is possible) across all survey areas. Space does not suffice for a full examination of the findings from each survey, although Table 1 provides a numeric overview of the number and proportion of the signs in the RL in the twenty survey areas from each part of France.

The data contributes to the large body of literature on the minority languages of France, not least since they illustrate the written use of languages in the public

Table 1. Quantitative results of signs in the RL

\begin{tabular}{lccl}
\hline Survey area & Total number of signs & Signs in the RL & Percentage \\
\hline Brittany & 10,950 & 44 & $0.4 \%$ \\
Corsica & 9,123 & 592 & $6.5 \%$ \\
Northern Catalonia & 9,645 & 118 & $1.2 \%$ \\
Marseille & 9,909 & 0 & $0 \%$ \\
Flemish France & 12,577 & 20 & $0.2 \%$ \\
Guadeloupe & 5,408 & 22 & $0.4 \%$ \\
Martinique & 7,038 & 14 & $0.2 \%$ \\
Nice with Monaco & 11,493 & 5 & $0.04 \%$ \\
New Caledonia & 6,169 & 1 & $0.02 \%$ \\
\hline
\end{tabular}


space, either transgressively or by those with both the authority and the ability to mark the walls of a city or town. The data furthermore point to the ways in which the regional languages are currently deployed. The first clear advantage of a quantitative approach to data collection is that it permits the cross-referencing between the use of Catalan, Guadeloupean creole, or Kanak for comparative and contrastive purposes. Comparisons between RLs are only tenable when the sole variable is the language. Whilst all the other parameters are identical, the fact that only the ethnolinguistic space changes between survey area means that we can argue securely that Xish, to employ Fishman's shorthand, assumes a wider role in the life of Xland than Yish does in Yland. This kind of generalization is less credible when the methods of recording data remain open to charges of impressionism or partiality, as is the case when the parameters for data collection are based on other criteria.

\subsubsection{The diachronic potential}

Pavlenko (2010) highlights the potential of the LL for providing indicators of language change (both in terms of the internal structure of language, and in attitudes towards a given language's use) in her reconstruction of Kyiv. Over a far shorter time period, this project points diachronically to trends in language practices from the specific perspective of minority language revitalization. The selection of survey areas, the recording of all signage in those specific areas, and the coding of signs according to several aspects lends itself to periodic returns in order to establish variation in the LL over a specific timeframe; in this case, we highlight the revisiting of Brittany and Northern Catalonia in 2014, following initial surveys in 2007 and 2008 respectively. The seven/eight year gap between surveys, during which time both survey areas have been exposed to different sociolinguistic pressures, including developments in language policies, migration of peoples, the transformation of ethnolinguistic and other social factors, and an economic shock, permit a close examination of local practices set against regional, national, and transnational change. As such, we return to the cities first examined in Blackwood (2010), Perpignan and Rennes, and underscore both the evolution in our own approach to LL fieldwork and the maturation of the public space as a site for language revitalization.

Space permits only a brief overview of the findings from this revisiting of Brittany and Northern Catalonia, but both second surveys highlight specific trajectories in terms of reversing language shifts. From the perspective of signs recorded in the RLs, most striking is the stability of Breton in Rennes in comparison with the changes in the visibility of Catalan in Perpignan. From the perspective of minority language revitalization, the negligible increase in the number of signs in Breton points to a stalled process of making the LL a 'domain of necessity' 
(Edwards, 2007:244). In total, only one sign in Breton recorded in 2007 had disappeared by 2014 , and this was a relatively ephemeral sticker in a shop window (analysed in greater detail in Blackwood, 2010:297). The explanation for the loss of this flash of Breton can only be conjectured - the possibility that the sticker disintegrated and was lost is as likely as it having been removed by someone ideologically opposed to the Breton-language school system which the sticker promoted. More conspicuous than the disappearance of a sticker is the appearance of two new street signs, one on the rue du Chapitre, the other on the rue SaintMelaine, both of which provide the name of the street in French above the version in Breton. For the rue Saint-Melaine, the sign (Figure 1) also includes information about Melaine, a sixth-century Bishop of Rennes. ${ }^{1}$

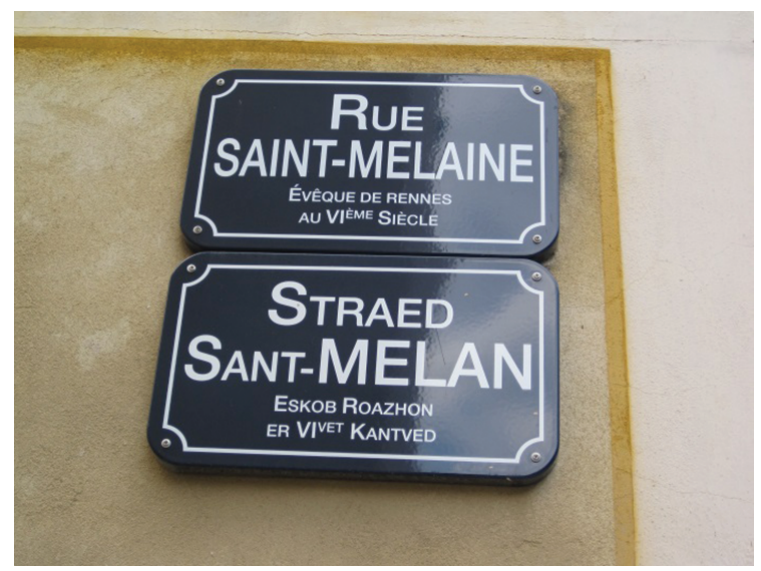

Figure 1. A bilingual street sign erected since 2008

The increase in the use of the RL by the city council is not numerically substantial, but in terms of wider significance, it is worth dwelling on this development. In February 2014, Rennes city council signed the Breton Language Charter, Ya d'ar Brezhoneg ('Yes to Breton'), and committed to level two compliance which - from the perspective of the LL - calls for the installation of bilingual street signs during renovations of the public space. This process is evident in two of the streets surveyed, and whilst insignificant quantitatively, the normalization of the RL is meaningful in terms of the civic authorities attitudes towards Breton. The only other addition in Breton to the public space recorded in 2014 was the inclusion of Breton in a welcome sign in the window of a driving school (Figure 2); remarkably, the Breton-language greeting appears beneath the French and Arabic text,

1. This approach echoes the configuration of street signs in French and Occitan dissected by Amos (forthcoming) but in a more consistent manner, where no extra information is given in either language. 
commenting as much on the saliency of Arabic in France's linguistic market as on the emplacement of the RL. According to the categorization proposed by Jaworski (2015), this sign might be qualified as part of what he calls the multilingual display frame. We argue that this sign reflects a lived multilingualism in Rennes, and so is possibly more appropriately understood as part of the spectacle frame, not least because its phatic function is, in Jaworski's terms, more likely to be viewed than read.

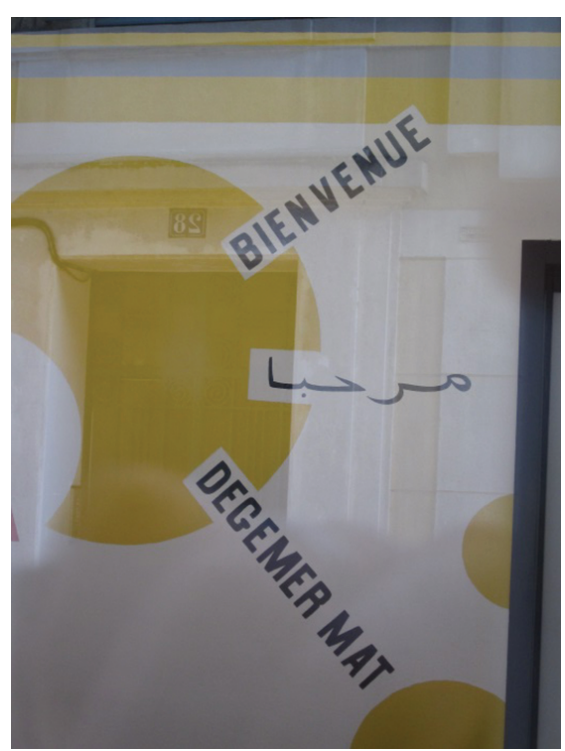

Figure 2. A trilingual welcome sign (in French, Arabic, and Breton) at a driving school in Rennes.

In Perpignan, over the six-year period between the two surveys, the composition of the LL has been more fluid, and there is a dynamism in the development of the public space that is not found in Rennes. On the one hand, Catalan has disappeared from parts of the survey areas in Perpignan, in response both to economic issues and public understanding of national language policy. On the other hand, and for the same two factors, there has been an increase in the visibility of the RL, meaning that there has been a net increase of 28 signs featuring Catalan. Quantitatively, the impact of this is slight, and so we turn to the analysis of this small sub-corpus to identify the reasons for this trend. The closure of the t-shirt shop Botiga Xot on rue Louis Blanc (to be replaced by a shop selling e-cigarettes) erases Catalan from that particular street; according to neighbouring tradesmen, the owners left in response to the economic downturn which began in 2008. At the same time, a comparable shop, Esperit Català, has opened within the survey area, on rue Mailly, which numerically replaces the 'lost signs' from the closure of 
Botiga Xot. Esperit Català also includes in its shop window a sign not recorded in 2008, which proudly claims Northern Catalonia for the provenance of the products (Figure 3). One of the defining characteristics of the produce on sale in this gift shop is the Franco-Catalan nature of the goods, of which the language on the t-shirts simultaneously authenticates the products as local, and commodifies the language for the purposes of consumption.

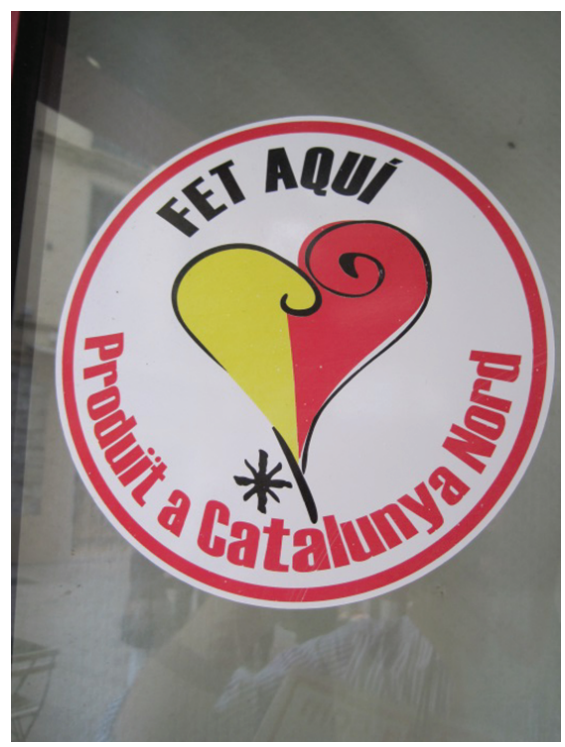

Figure 3. 'Made in Northern Catalonia' sticker

The other step change in the revitalization of Catalan in the LL attested by this diachronic approach to data collection is the notable role played by the civic authorities who increasingly emplace the RL in the public space and are, according to the 2014 survey, the key agent in the normalization of Catalan in Perpignan. What is striking here is that it is the elected council, as the local embodiment of the State, who are investing in signage in the built environment that not only concedes space to Catalan, but in some examples privileges Catalan over French. At one level, the inclusion of Catalan in the information panels on ticket machines at bus stops, or on the display boards dotted around the city commenting on aspects of architectural note, echoes the current phase of language management in France's regions, whereby the RL is allocated space alongside French. More arresting, in the light of centuries of an unwavering language ideology, is the production of monolingual street signs in the RL, as attested on the avenue du Général de Gaulle. That Perpignan city council name the street which honours the twentieth-century embodiment of the French Fifth Republic and all its ideological implications in Catalan rather than French is at the very least ironic. 


\subsection{The challenges of a quantitative approach}

\subsubsection{The paucity of signs in the Regional Languages}

At first glance, the overview of the nine corpora in Table 1 highlights the striking paucity of signs in the RLs. When discussing the visibility or otherwise of RLs in France's LL, we are of course talking in relative terms. As has been attested in all of our published findings thus far, the French language dominates the LL to the extent that other languages, be they the RLs, English, or the languages of migrant groups in France, are statistically marginalized. Across the surveys, French appears on average in three quarters of the signs recorded, and centuries of strident language management strategies to ensure the hegemony of French as a written language for France, even in spaces as disparate as those studied here, clearly have had an impact. That the French language is dominant in France and her territories as a consequence of language policies is a finding reported widely in research published over the last century, and therefore this perspective is not particularly fruitful. Where there is the potential for LL research to add to the wider understanding of language practices in France is the symbiotic approach, which we discuss in the next section.

The rarity of signs in the RL is most notable in Marseille where no signs featuring either Provençal or Occitan are recorded. The complex nature of the relationship between the city of Marseille and RLs is something we discuss more fully elsewhere (Blackwood and Tufi, 2015), but in this discussion, it suffices to note the connection between Provençal or Occitan and France's second city is - for a variety of reasons - much looser than the affiliation between somewhere like Lille and Flemish, or Rennes and Breton, or Perpignan and Catalan. This is not to say, however, that there were no examples of a RL visible anywhere in Marseille; indeed, on the rue des Trois Mages, in Marseille's second arrondissement, is the Occitan Cultural Centre, featuring Occitan - a sister variety of Provençal - in several signs, but this is not in one of the streets surveyed. This glaring limitation to a quantitative approach to LL research that is grounded in the selection of a definitive number of survey areas is something to which we return below when we consider alternative and supplementary approaches to exploring the visibility of RLs in France's public space.

\section{Potential benefits of a symbiotic approach to data collection}

In the light of the challenges posed by a quantitative approach highlighted in the section above, we propose a mixed economy for data collection for LL research into the revitalization of RLs. This symbiosis is predicated on the understanding 
that a qualitative approach on its own cannot provide either the diachronic or the comparative aspects that characterize the quantitative approach. Without the statistical foregrounding that permits comparison and facilitates an exploration of change over time, quantitative studies run the risk of appearing decontextualized and, therefore, distorted. The counting and coding of signs from within definable survey areas permit a close examination of language use along a number of vectors, including function. It is this angle that, once contextualized by a statistical overview, enables a close analysis and better understanding of the ways in which RLs are used across France today. When we consider the findings in Table 1 from the perspective of the primary function of each sign, a pattern emerges, highlighting that the two languages proportionally most visible within their respective LLs are those where the language often assumes a symbolic role, as identified by the category 'legend'. For this project, we understand a legend as an inscription of a wide variety of kinds which includes slogans, trade association membership stickers, and text which does not seek to instruct or inform (including more lyrical or poetic text), all of which coalesce to contribute to the symbolic construction of multilingualism in France.

To this end, the practices discerned on Corsica echo the findings of Pietikäinen and Kelly-Holmes (2011:325) who argue that the attestations of language discussed here 'act as advertisements for the place and provide an element of differentiation and authentification between 'there' and elsewhere, between that particular heritage and other cultures, and between locally-made and elsewhere-made'. In this case, the opposition is framed around the poles of Corsica as the authentic Other and France (in a broad sense) as elsewhere. The added economic value attributed by the island's manufacturers, as well as those who hold franchises for national or international firms, points to the commodification of Corsican in ways not replicated as consistently and as thoroughly elsewhere in France. This use of the RL to carve out a space in the LL for Corsican is more widespread than, for example, brand names or labels on products, although examples of these categories are also attested in the survey areas. Whilst we find honey with bilingual labels (discussed in Blackwood \& Tufi, 2012), cola drinks with both the RL and French on the cans (see Blackwood, forthcoming), and even the name of a bus line in Corsican (Blackwood, 2014), the value of linguistic resources in the RL is ideologically charged as simultaneously opposing the hegemonic French language and localizing material culture in the island's differentiated identity.

This pattern is replicated, although not quite so strikingly, in Northern Catalonia, where Catalan is commodified in the linguistic market, again often as a marker of authenticity. The use of Catalan in legends is complemented by culturally salient colours (the red and yellow of Catalan cultural identity) in ways not reproduced on Corsica. The economic attractiveness of Catalan continues into 
businesses such as the travel agency Catalunya Evasió which is named by its French owners in Catalan to address, in their words, a Catalanophone clientele. Having established that this use of the RL is the most widespread in Northern Catalonia, we can conclude that the LL contributes to the construction of the Catalan-ness of this part of France, and commodifies that characteristic through language which can be consumed - i.e. purchased - and distributed.

This exploration along functional lines complements the frames approach proposed by Kallen (2010). He proposes the reconceptualization of the LL as 'a confluences of systems, observable within a single visual field but operating with a certain degree of independence between elements' in order to highlight the relationship between areas of the site of inquiry. Eschewing a quantitative approach, Kallen's visual frames invite the researcher to identify a key area of shared community life and explore the relationship between languages in this partial aspect of the wider public space. Thus far, we have applied this frames approach on Corsica (Blackwood, 2014), focussing in particular on the civic frame in order to differentiate between the actions of the State, the regional authorities, and the town council in Ajaccio to tease out the differences in language management. This is not a quantitative approach in the manner of the earlier studies, but is instead based on discussing signs placed by each of the three different civic authorities, concluding that there are strikingly different approaches, ranging from a complete absence of Corsican in spaces governed by the State, whilst the regional authorities, explicitly tasked with extending the use of Corsican, fail to exploit all the opportunities of the spaces they control. It is at the local level in Ajaccio, where the town council oversees signage, that the widest use of Corsican is attested. There is no counting of signs in this approach, and the aim is to be as exhaustive as possible in the specific frame under examination. Nevertheless, this kind of study is contextualized by the quantitative approaches that have preceded it.

\section{Conclusions}

On the basis of eight years of LL fieldwork in France, it is clear that a hybrid approach to research in languages in the public space, especially when focussing on attempts to reverse a language shift, is both viable and necessary. From the perspective of language revitalization, when discussing France's RLs, a quantitative approach, despite its shortcomings outlined above, is particularly helpful for cross-referencing over a large geographic space. The fact that the only variable in terms of data collection is the RL of the ethnolinguistic group permits the kind of analysis that identifies a trend towards the symbolic use of Corsican and Catalan in ways not replicated for Flemish or Nissart. In a territory that covers 
three continents, quantitative data collection as explored here, and the subsequent analysis, resist the potential for silofication. At the same time, given the paucity of signs in the RLs, a qualitative dimension is imperative, since it permits the privileging of certain frames whilst the analysis remains grounded in contexts drafted in part by quantitative discussions. Above all, the diachronic potential of this symbiotic approach is an ideal methodology for generalizing about trends in language revitalization without recourse to impressionism or anecdotes. As highlighted by the findings in Rennes and Perpignan, the LL is paradoxically both fluid and fixed. Changes in the ownership of premises, the ephemeral nature of some signs, the evolution in language beliefs, and economic trajectories all contribute to the variability of the public space over a relatively short period of time. Unexpectedly, the data collected points to only the slightest of changes in the visibility of Breton, and a marginally more noteworthy evolution in the emplacing of Catalan in the LL.

If this dual approach merely led to the inference that French is visually dominant in France's public space, it would be difficult to argue convincingly that quantitative and qualitative research offer much more than the analysis of the same signs but from different perspectives. However, we would argue that using both quantitative and qualitative approaches provides the most comprehensive overview of language use in France. This marriage of two methodologies - often divorced from one another in LL research - is significant since the conclusions to which, in combination, they point are greater than the sum of their parts. Having started with a statistical approach, it is possible to examine more closely the function, authorship, materiality, and target audiences of signs in the RLs. This reveals, for example, in Perpignan the counter-intuitive trend whereby the civic authorities are emerging as the key agent in minority language revitalization. In a country famed for its unwavering top-down support for the national standard language, it is the local authorities, using income generated through taxation, who are at the forefront of reversing the language shift in Northern Catalonia. Their commitment to providing information, be that details on notable architectural features or the names of streets, in the RL far outstrips the emplacement of Catalan in product labelling, in business names, or in graffiti. At the same time, and at the other end of the country, there is little evidence to suggest a transformation in the use of Breton in the LL. This is all the more conspicuous given the commitment of activist groups to the wider visibility of the RL across Brittany; the surveys of 2007 and 2014 suggest very little practical change to a cityscape that might include other Breton material culture, including flags, colours, and various symbols, but which is barely marked by the Breton language.

These two mini case studies highlight the potential for the combined methodology to facilitate comparison across a wide space, and to identify change over a relatively short but significant period of time. Conclusions drawn from these 
are grounded in a stable, convincing quantitative approach whose statistical data is re-evaluated qualitatively in the ways recognized in this article. The relatively restricted datasets, contextualized by quantitative evaluations, are then explored qualitatively to point to new interpretations which we argue are not possible to reach without this synergetic approach. We therefore conclude not by insisting that all further scholarship into the LL is undertaken according to this dual methodology. However, we hope that research underpinned by quantitative starting points, supplemented by close qualitative analysis, will no longer be dismissed as merely "counting signs".

\section{References}

Amos, H. W. (Forthcoming). Redefining Multilingual Signs in the Linguistic Landscape: Discursive content and text-types on street signs in Toulouse.

Backhaus, P. (2007). Linguistic landscapes: A comparative study of urban multilingualism in Tokyo. Clevedon: Multilingual Matters.

Barni, M., \& Vedovelli, M. (2012). Linguistic landscapes and language policies. In C. Hélot, M. Barni, R. Janssens, \& C. Bagna (Eds.), Linguistic landscapes, multilingualism and social change (pp. 27-38). Frankfurt: Peter Lang.

Ben-Rafael, E., Shohamy, E., Amara, M.H., \& Trumper-Hecht, N. (2006). Linguistic landscape as symbolic construction of the public space: The case of Israel. International Journal of Multilingualism, 3(1), 7-30. DOI: 10.1080/14790710608668383

Blackwood, R. (2010). Marking France's public space: Empirical surveys on regional heritage languages in two provincial cities. In E. Shohamy, E. Ben-Rafael, \& M. Barni (Eds.), Linguistic landscape in the city (pp. 292-306). Clevedon: Multilingual Matters.

Blackwood, R. (2014). The top-down revitalisation of Corsican: Considering the reversal of a language shift in the linguistic and semiotic landscapes of Ajaccio. French Studies, 68(1), 61-77. DOI: $10.1093 / \mathrm{fs} / \mathrm{knt} 224$

Blackwood, R. (Forthcoming). Chestnut beer, Corsica-Cola, and wine bottles: The commodification of Corsican in the linguistic and semiotic landscapes of the island's drinks industry. International Journal of the Sociology of Language.

Blackwood, R., \& Tufi, S. (2012). Policies vs. non-policies: Analysing regional languages and the national standard in the linguistic landscape of French and Italian mediterranean cities. In D. Gorter, H.F. Marten, \& L. van Mensel (Eds.), Minority languages in the linguistic landscape (pp. 109-126). Houndmills: Palgrave Macmillan.

Blackwood, R., \& Tufi, S. (Forthcoming). The linguistic landscape of the Mediterranean: French and Italian coastal cities. Houndmills: Palgrave Macmillan.

Edwards, J. (2007). Back from the brink: The revival of endangered languages. In M. Helinger \& A. Pauwels (Eds.), The handbook of language and communication: Diversity and change (pp. 241-269). Berlin: Mouton de Gruyter.

Gorter, D. (Ed.). (2006). Linguistic landscape: A new approach to multilingualism. Clevedon: Multilingual Matters. 
Jaworski, A. (2015). Welcome: Synthetic personalization and commodification of sociability in the linguistic landscape of global tourism. In B. Spolsky, O. Inbar-Lourie, \& M. Tannenbaum (Eds.), Challenges for language education and policy: Making space for people (pp. 214-231). London: Routledge.

Kallen, J. (2010). Changing landscapes: Language, space and policy in the Dublin linguistic landscape. In A. Jaworski \& C. Thurlow (Eds.), Semiotic landscapes: Language, image, space (pp. 41-58). London: Continuum.

Landry, R., \& Bourhis, R. (1997). Linguistic landscape and ethnolinguistic vitality: An empirical study. Journal of Language and Social Psychology, 16, 23-49.

DOI: 10.1177/0261927X970161002

Malinowski, D. (2009). Authorship in the linguistic landscape: A multimodal-performative view. In E. Shohamy \& D. Gorter (Eds.), Linguistic landscape: Expanding the scenery (pp. 107-125). London: Routledge.

Milon, A. (2002) Tags and murals in France: A city's face or natural landscape. In A.-P. Durand (Ed.), Black, blanc, beur: Rap music and hip-hop culture in the Francophone world (pp. 8798). Lanham, MD: The Scarecrow Press.

Pavlenko, A. (2010). Linguistic landscape of Kyiv, Ukraine: A diachronic study. In E. Shohamy, E. Ben-Rafael, \& M. Barni (Eds.), Linguistic landscape in the city (pp. 133-150). Clevedon: Multilingual Matters.

Pennycook, A. (2010). Language as a local practice. London: Routledge.

Pietikäinen, S., \& Kelly-Holmes, H. (2011). The local political economy of languages in a Sami tourism destination; Authenticity, mobility in the labelling of souvenirs. Journal of Sociolinguistics, 15(3), 323-346. DOI: 10.1111/j.1467-9841.2011.00489.x

Saez Rivera, D., \& Castillo Lluch, M. (2012). The human and linguistic landscape of Madrid (Spain). In C. Hélot, M. Barni, R. Janssens, \& C. Bagna (Eds.), Linguistic landscapes, multilingualism and social change (pp. 309-328). Frankfurt: Peter Lang.

Stroud, C., \& Mpendukana, S. (2009). Towards a material ethnography of linguistic landscape: Multilingualism, mobility and space in a South African township. Journal of Sociolinguistics, 13(3), 363-386. DOI: 10.1111/j.1467-9841.2009.00410.x

\section{Author's address}

Robert Blackwood

Department of Modern Languages

\& Cultures

University of Liverpool

Cypress Building

Chatham Street

Liverpool, Merseyside L69 7ZR

UK

Robert.Blackwood@liverpool.ac.uk 